Check for updates

Cite this: RSC Adv., 2017, 7, 26113

Received 7th December 2016

Accepted 10th May 2017

DOI: 10.1039/c6ra27873f

rsc.li/rsc-advances

\section{A graphene quantum dot/phthalocyanine conjugate: a synergistic catalyst for the oxygen reduction reaction $\uparrow$}

\author{
Ki Hwan Koh, ${ }^{a}$ Sung Hyun Noh, ${ }^{a}$ Tae-Hyun Kim, ${ }^{b}$ Won Jun Lee, (D) ${ }^{c}$ Sung-Chul Yi ${ }^{\star b}$ \\ and Tae Hee Han iD *a
}

Graphene quantum dots (GQDs) have gained great interest due to their chemical stabilities, unique catalytic activities, and feasible chemical functionalization opportunities; these have opened up new applications in the fields of chemical energy conversion and storage. Herein, we synthesized iron(I) phthalocyanine (FePC)-GQD conjugates as facile electrocatalysts for the enhanced oxygen reduction reaction (ORR), which is critical in energy conversion systems of fuel cells. This unique combination of materials (GQDFePC) exhibits a greatly enhanced onset potential via a four-electron pathway in an alkaline electrolyte. Moreover, the synthesized electrocatalyst shows distinguished tolerance toward methanol and carbon monoxide, which paves the way for its commercialization as an electrocatalyst.

\section{Introduction}

Graphene-based quantum dots (GQDs) have been extensively investigated due to their various advantages including easy synthesis procedures, non-toxicity, chemical/physical stability, and controllable chemical functionality. ${ }^{1}$ Interestingly, GQDs can significantly lower the oxygen adsorption barrier and the first electron transfer barrier, and provide enhanced electrocatalytic activity for the oxygen reduction reaction (ORR) due to the increased portion of electrocatalytically active edge sites. ${ }^{2}$ Therefore, GQDs are gathering huge research interest as an efficient alternative to Pt-based electrocatalysts for the ORR. ${ }^{3}$ However, challenges such as low limiting current density and onset potential still exist. ${ }^{4}$ Of course, to compete with Pt-based catalysts, the intolerance to fuel crossover from the anode, ${ }^{5}$ deactivation by $\mathrm{CO}$ poisoning effect, and electrochemical stability, which is often found in the conventional catalysts, should be overcome. ${ }^{6}$

The hybridization of GQDs with other functional materials represents a potential strategy that can be used to improve their catalytic properties. ${ }^{7}$ In particular, iron(II) phthalocyanine (FePC) is one of the promising nanomaterials among nonprecious-metal category that can be used as an

${ }^{a}$ Department of Organic and Nano Engineering, Hanyang University, 222 Wangsimni-Ro, 04763, Seoul, Republic of Korea. E-mail: than@hanyang.ac.kr

${ }^{b}$ Department of Chemical Engineering, Hanyang University, 222 Wangsimni-Ro, 04763, Seoul, Republic of Korea.E-mail: scyi@hanyang.ac.kr

${ }^{c}$ Department of Chemistry, Imperial College London, London, SW7 2AZ, UK

$\dagger$ Electronic supplementary information (ESI) available: A schematic conjugation process of GQD and FePC, SEM images of FePC and magnified FePC. See DOI: $10.1039 /$ c6ra27873f electrocatalyst for the ORR. ${ }^{8-11}$ FePC has a conjugated macrocyclic structure with azo and pyrrolic nitrogen atoms. ${ }^{12}$ Therefore, conjugation with GQDs would be beneficial for enhancing the electrocatalytic performance. In addition, as demonstrated with volcano theory, the introduction of nitrogen in graphene materials is beneficial to enhance the electrocatalytic performance for ORR..$^{13,14}$ Doped nitrogen increases the charge density of graphene and results in a better affinity of graphene with oxygen and an enhanced ability to weaken the $\mathrm{O}-\mathrm{O}$ bonding on the surface of catalysts, indicating the reduced overpotential, and better electrocatalytic performance for ORR. ${ }^{15}$

In this work, we covalently functionalized GQDs with FePC by a facile ferric chloride reaction in order to improve the ORR performance. Electrochemical measurements showed that GQDs functionalized with iron phthalocyanine (GQD-FePC) exhibited enhanced electrocatalytic activity with an electron transfer number of 3.8 in an alkaline medium; this indicates a direct four-electron pathway to $\mathrm{OH}^{-}{ }^{16} \mathrm{GQD}-\mathrm{FePC}$ also showed great tolerance to methanol crossover and the $\mathrm{CO}$ poisoning effect compared with a commercial PlatinumCarbon (Pt/C) catalyst, which suggests that GQD-FePC could be used as an alternative electrocatalyst to commercial Pt/C in fuel cells.

\section{Experimental}

\section{Materials}

$\mathrm{Pt} / \mathrm{C}$ was purchased from Alfa Aesar (platinum, nominally 20\% on carbon black, HiSPECTM 3000). FePC, ammonium hydroxide solution (30\%), and ethanol (99.5\%) were purchased from Sigma-Aldrich. Hydrogen peroxide (Guaranteed Reagent, 99.3\%) and dimethyl sulfoxide (DMSO, Guaranteed Reagent, 
99\%) were purchased from Junsei. No further purification was done, and all chemical were used as received.

\section{Preparation of GQDs, and conjugate of GQD with FePC}

Graphene oxide (GO) (400 mg) was first prepared using the modified Hummers' method, as reported previously. ${ }^{17}$ Then, $400 \mathrm{mg}$ of GO powders were dispersed in a $200 \mathrm{~mL}$ solution of $\mathrm{NH}_{4} \mathrm{OH} / \mathrm{H}_{2} \mathrm{O}_{2} / \mathrm{DIW}(1: 2: 20$ by volume). This solution then underwent a hydrothermal treatment in a closed vessel at $150{ }^{\circ} \mathrm{C}$ for $5 \mathrm{~h}$. The final product (GQDs powder) was obtained after freeze-drying. ${ }^{\mathbf{1 7}}$ GQD-FePC was synthesized by a ferric chloride test reaction. For this, $9 \mathrm{mg}$ of GQDs, $1 \mathrm{mg}$ of FePC, and $10 \mu \mathrm{L}$ of pyridine were added into $10 \mathrm{~mL}$ of DMSO. This mixture was stirred and reacted for $20 \mathrm{~min}$ at room temperature. During the reaction, the mixture colour was changed from blue to green. Conjugated materials were obtained by freeze-drying after washing.

\section{Characterization}

Morphologies of the GQDs, FePC aggregates, and GQD-FePC conjugated materials were characterized via transmission electron microscopy (TEM; JEOL, JEM-2100F) operated at an accelerating voltage of $200 \mathrm{kV}$. TEM samples were prepared on lacycarbon TEM grids (Ted Pella, Inc.). X-ray photoelectron spectroscopy (XPS) measurements were performed with an XPS spectrometer (Thermo Fisher Scientific, Theta probe) using a monochromic $\mathrm{Al} \mathrm{K} \alpha$ radiation X-ray source. Fourier-transform infrared spectroscopy (FT-IR) analyses were carried out using a Thermo Fisher Scientific Co. Nicolet 6700 with $\mathrm{KBr}$ pellets. A UV-vis spectrometer (Analytik Jena, Specord200) was used to obtain the UV-vis absorption between 300 and $800 \mathrm{~nm}$ at a resolution of $1 \mathrm{~nm}$.

\section{Electrochemical measurements}

Electrochemical analysis, including cyclic voltammetry (CV), linear sweep voltammetry (LSV), and chronoamperometry (used to evaluate the ORR activity and stability of the prepared catalysts), were carried out using a computer-controlled potentiostat (SP-200, Bio-Logic SAS instrument, France) with a typical threeelectrode cell possessing a gas flow system; these tests were performed in an $\mathrm{O}_{2}{ }^{-}$saturated $0.1 \mathrm{M} \mathrm{KOH}$ electrolyte at $30^{\circ} \mathrm{C}$. A platinum wire and $\mathrm{Hg} / \mathrm{HgO}$ were used as the counter electrode and the reference electrode, respectively. The working electrodes were prepared by drop-casting $10 \mu \mathrm{L}$ of GQDs and GQDFePC suspensions in DMSO $\left(1 \mathrm{mg} \mathrm{mL}^{-1}\right)$ onto the glassy carbon electrode surface (GC, $5 \mathrm{~mm}$ diameter, Pine Instrument Co.). The electrodes were dried in a vacuum oven at $90{ }^{\circ} \mathrm{C}$ for $6 \mathrm{~h}$, treated with $5 \mu \mathrm{L}$ of a $5 \mathrm{wt} \%$ Nafion solution, and dried in ambient conditions. For a comparison with commercial $\mathrm{Pt} / \mathrm{C}$ catalysts for the ORR, a Pt/C catalyst ink was also prepared in ethanol $\left(1 \mathrm{mg} \mathrm{mL}{ }^{-1}\right)$. Then, $10 \mu \mathrm{L}$ of the Pt/C catalyst ink was introduced onto the glassy carbon electrode surface. The electrode was subsequently dried at ambient conditions and then coated with a Nafion solution. The rotating disk electrode (RDE) test was performed with an electrode rotator (modulated speed rotator, Pine Instrument Co.).

\section{Results and discussion}

The schematic structures of GQDs and GQD-FePC are illustrated in Fig. 1. The GQDs synthesized by the hydrothermal reaction are naturally decorated with the oxygen- and nitrogencontaining functional groups, including carbonyl, hydroxyl, amine, and amide groups, ${ }^{17}$ as shown Fig. 1a. These nitrogen atoms, which are chemically bonded with carbon atoms, not only influence the optical properties of GQDs, but also alter the electronic characteristics by contributing more electrocatalytic active sites. ${ }^{4}$ In addition, the oxygen-containing functional groups of GQDs provide reaction sites for hybridization with other nanomaterials. ${ }^{18}$ In this study, GQDs were functionalized with FePC in order to take advantage of the synergistic effects on the ORR activity. Conjugation of GQDs with FePC (Fig. 1b) was readily achieved by the ferric chloride reaction, as detailed in Fig. S1. $\dagger$ The morphologies of the GQDs, FePC, and GQDs-FePC were analyzed by TEM. Fig. 1c shows the appearance of GQDS with an average lateral size of $2.76 \mathrm{~nm}$ and a typical GQD morphology. This zero-dimensional structure provides much more electrocatalytic active sites than the larger graphene due to the charge polarization of edge carbon atoms. ${ }^{2}$ However, FePC molecules formed anisotropic aggregates (Fig. 1d and

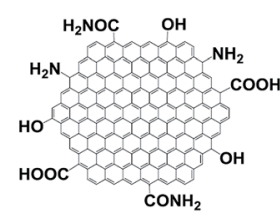

C

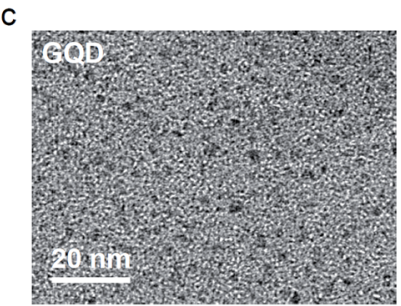

e

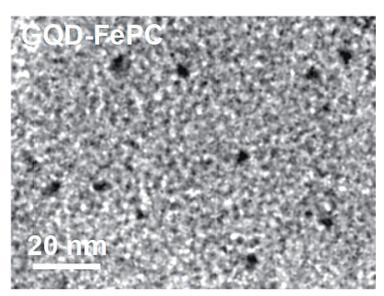

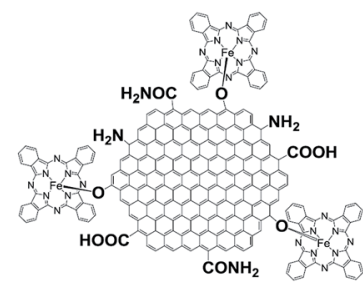

d

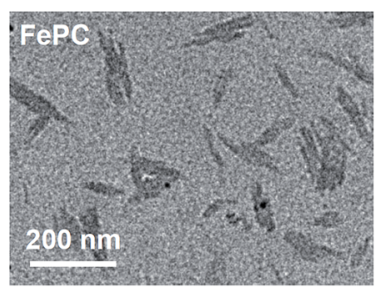

f

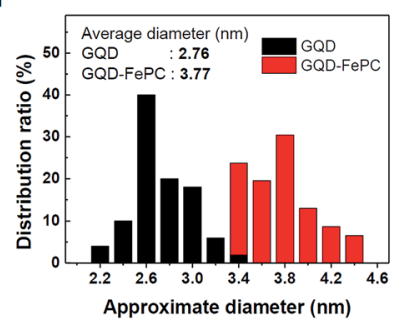

Fig. 1 Structural illustrations of (a) GQDs and (b) GQD-FePC compounds. TEM images of (c) GQD, (d) FePC, and (e) GQD-FePC. GQDs show the dot-like shape, while anisotropic aggregates were observed in FePC due to the self-assembly of FePC. As FePC was conjugated successfully with GQDs, dot-like morphology was mainly shown in GQD-FePC. (f) Size distribution of GQDs and GQD-FePC. The average diameters of the GQDs and GQD-FePC are 2.76 and $3.77 \mathrm{~nm}$, respectively. 
$\mathrm{S} 2 \dagger)$. This is caused by the self-assembly of FePC. This assembly behaviour induces an inevitable decrease in the active site area, which eventually results in performance degradation. ${ }^{19-21}$ Interestingly, the GQD-FePC conjugates (Fig. 1e) did not have rod-shaped morphologies, but mainly showed dot-like shapes as the representative morphology. As shown in Fig. 1f, the size of the hybrid materials was slightly increased to $3.77 \mathrm{~nm}$ after conjugation, indicating the successful hybridization and molecular-level dispersion of GQDs and the hybrid form.

FT-IR spectra were measured to investigate the chemical properties of GQDs, FePC, and GQD-FePC. As shown in Fig. 2a, the five major peaks of GQDs (black line) appeared at 1335, 1402, 1436,1606 , and $1766 \mathrm{~cm}^{-1}$; these correspond to the vibration modes of $\mathrm{C}-\mathrm{N}$ stretching (amine and amide) and $\mathrm{C}=\mathrm{O}$ stretching (amide and carboxylic acid), respectively. ${ }^{17}$ Theses typical peaks of GQDs indicate that the as-prepared GQDs already possess several types of the nitrogen-containing functional groups. In the case of FePC (red line), the presence of strong peaks was observed at 732 $\mathrm{cm}^{-1}$ (for $\mathrm{C}-\mathrm{H}$ out-of-plane bending vibration), $754 \mathrm{~cm}^{-1}$ (for benzene and isoindole in-plane deformation), and $781 \mathrm{~cm}^{-1}$ (for Fe-N stretching). ${ }^{22}$ Other representative peaks of FePC were observed at 1079, 1120,1332, and $1513 \mathrm{~cm}^{-1}$. These were assigned to $\mathrm{C}-\mathrm{N}$ stretching, $\mathrm{Fe}-\mathrm{N}$ stretching (pyrrole), $\mathrm{C}=\mathrm{N}$ stretching (pyrrole rings), and isoindole stretching (in the plane of the FePC macrocycle), respectively. ${ }^{22}$ These major peaks of FePC were also present in the GQD-FePC hybrid materials (blue line), indicating the additional introduction of nitrogen into the GQDs.

To directly elucidate the bonding properties of GQD-FePC, XPS was employed. The high-resolution Fe 2p spectra of FePC
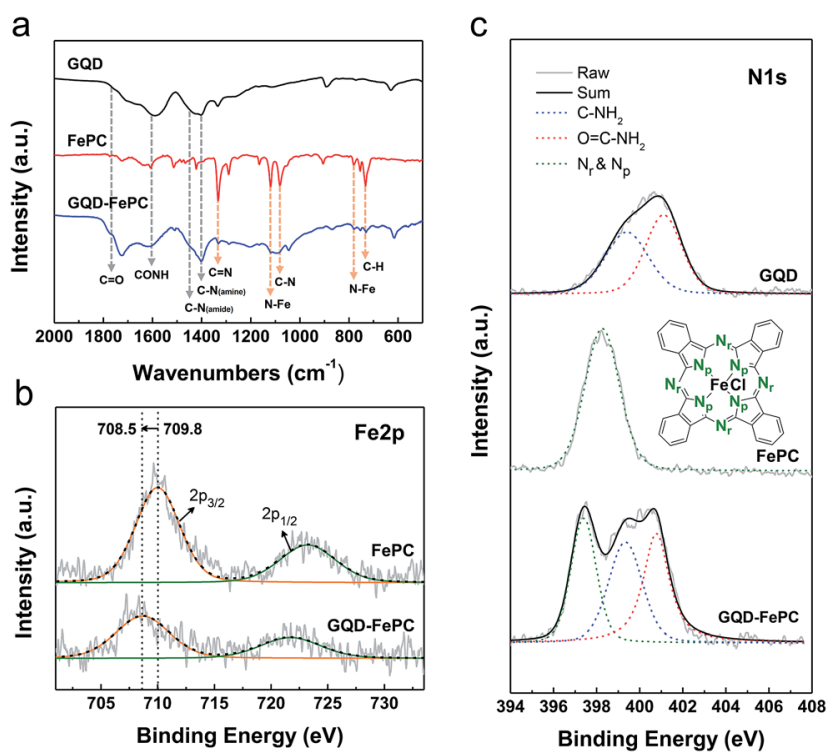

Fig. 2 (a) FT-IR spectra of GQDs, FePC and GQD-FePC. The major peaks of GQDs and FePC were observed in GQD-FePC spectra, indicating the successful conjugation between GQD and FePC. (b) High-resolution Fe $2 p$ peaks of FePC and GQD-FePC. (c) Highresolution $\mathrm{N}$ 1s XPS peaks of GQDs, FePC, and GQD-FePC; the amine group $\left(-\mathrm{NH}_{2}\right)$ is the blue dashed line, the amide group $(-\mathrm{CNHO})$ is the red dashed line, and the azo and pyrrolic nitrogen $\left(N_{r}\right.$ and $\left.N_{p}\right)$ are the green dashed line. $N_{r}$ and $N_{p}$ were highlighted with green color in a FePC molecule.
Table 1 Atomic percentage (atm\%) and components of $\mathrm{N}$ 1s in the GQDs, FePC, and GQD-FePC based on the XPS analysis

\begin{tabular}{lcl}
\hline Name & $\mathrm{N} 1 \mathrm{~s}(\mathrm{~atm} \%)$ & Components of $\mathrm{N} 1 \mathrm{~s}$ \\
\hline GQDs & 9.76 & Amine $\left(\mathrm{C}-\mathrm{NH}_{2}\right)$, amide $\left(\mathrm{O}=\mathrm{C}-\mathrm{NH}_{2}\right)$ \\
FePC & 17.75 & Azo $\mathrm{N}\left(\mathrm{N}_{\mathrm{r}}\right)$, pyrrolic $\mathrm{N}\left(\mathrm{N}_{\mathrm{p}}\right)$ \\
GQD-FePC & 11.56 & Amine $\left(\mathrm{C}-\mathrm{NH}_{2}\right)$, amide \\
& & $\left(\mathrm{O}=\mathrm{C}-\mathrm{NH}_{2}\right)$, azo $\mathrm{N}\left(\mathrm{N}_{\mathrm{r}}\right)$, pyrrolic $\mathrm{N}\left(\mathrm{N}_{\mathrm{p}}\right)$
\end{tabular}

and GQD-FePC are presented in Fig. $2 \mathrm{~b}$. The $\mathrm{Fe} 2 \mathrm{p}_{3 / 2}$ core level spectrum of FePC has a peak at $709.8 \mathrm{eV}$. After conjugation, the Fe 2p peak was shifted to a lower binding energy of $708.5 \mathrm{eV}$. This shift is due to the altered electronic structure after the formation of GQD-FePC owing to electron transfer behaviour. ${ }^{23}$ Among a variety of metal-cored phthalocyanine molecules, FePC shows a relatively strong electron withdrawing ability. ${ }^{24}$ Therefore, after conjugation with GQDs, the more electrons transfer from electron-rich GQD to $\mathrm{FePC}^{25}$ and it induces the charge density gradient between GQD and FePC. ${ }^{15}$ As being often observed when graphene is doped with nitrogen, this type of charge transfer narrows the bandgap of GQDs and subsequently improves the electrocatalytic activity of GQDs. ${ }^{15,26}$ In the narrow scan of the $\mathrm{N}$ 1s spectra of GQDs, FePC, and GQD-FePC (Fig. 2c), various nitrogen functional groups are shown. Three representative nitrogen functional groups in Fig. 2c show the amine group (blue line), amide group (red line), and azo and pyrrolic nitrogen of FePC (green line). ${ }^{27}$ The $\mathrm{N}$ 1s spectrum of GQDs was deconvoluted into amine and amide groups centered at 399.4 and $300.6 \mathrm{eV},{ }^{17}$ respectively, while only azo and pyrrolic nitrogen were observed in FePC. ${ }^{22}$ After the functionalization of GQDs with FePC, all of the nitrogen types were observed in the $\mathrm{N}$ 1s spectrum of GQD-FePC. In addition, as can be observed in the $\mathrm{N}$ 1s spectra of FePC and GQD-FePC, the peaks of azo and pyrrolic nitrogen for GQD-FePC were shifted to lower values by $0.9 \mathrm{eV}$ compared with those of FePC; this follows the same principles as the Fe 2 p peak shift. Detailed information about the $\mathrm{N}$ 1s spectra, including the atomic percentage and the components of each material, is summarized in Table 1. When GQDs were functionalized with FePC, an obvious increase in the $\mathrm{N}$ 1s atomic percentage (from $9.76 \%$ to $11.56 \%$ ) was directly observed. Based on the XPS characterization, the GQD-FePC hybrid materials have an average of 2.3 FePC molecules per GQD molecule.

The electrocatalytic activity of the GQDs and GQD-FePC catalysts for the ORR was examined by $\mathrm{CV}$ in a $0.1 \mathrm{M} \mathrm{KOH}$ electrolyte solution saturated with either $\mathrm{N}_{2}$ or $\mathrm{O}_{2}$, as shown in Fig. 3. For comparison, a Pt/C electrode was also prepared and tested under the same conditions. As shown in Fig. 3a-c, weak cathodic current peaks for reduction are observed for all samples in the $\mathrm{N}_{2}$-saturated electrolyte; this is attributed to the intrinsic redox peaks. ${ }^{19}$ In contrast, obvious cathodic current peaks are observed in the $\mathrm{O}_{2}$-saturated electrolyte, indicating the abilities of each catalyst to electrochemically catalyze the ORR. For the GQD catalyst, the ORR peak appears at $-0.18 \mathrm{~V} v s$. $\mathrm{Hg} / \mathrm{HgO}$. After the functionalization of GQDs with FePC, the 
a

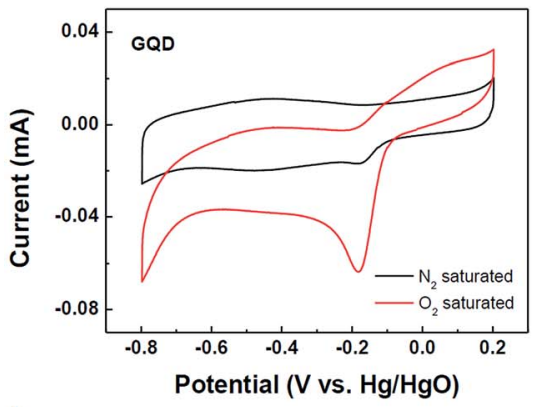

b

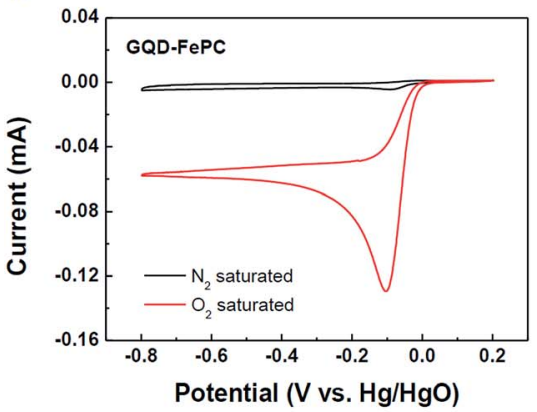

C

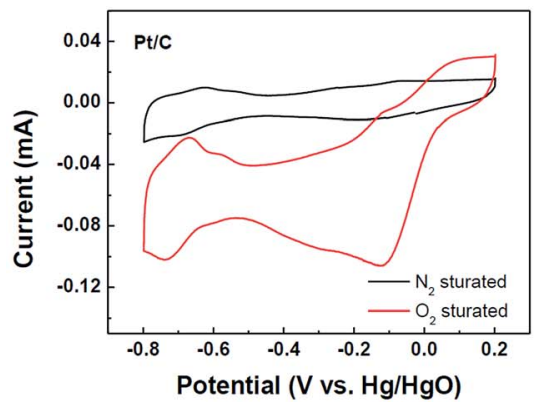

Fig. $3 \mathrm{CV}$ curves of (a) GQDs, (b) GQD-FePC, and (c) Pt/C in $\mathrm{N}_{2}$ saturated (black line) and $\mathrm{O}_{2}$-saturated (red line) $0.1 \mathrm{M} \mathrm{KOH}$ at a scan rate of $20 \mathrm{mV} \mathrm{s}^{-1}$. Small current peaks for redox in $\mathrm{N}_{2}$-saturated electrolyte are caused by the intrinsic redox of all samples. After the conjugation, the ORR peak of GQD-FePC positively shift to $0.1 \mathrm{~V}$ vs. $\mathrm{Hg} / \mathrm{HgO}$ and its oxygen reduction current was measured to be -0.13 $\mathrm{mA}$ which is over two times larger than that of pure GQDs.

ORR peak of GQD-FePC positively shifts to $-0.1 \mathrm{~V} v s$. $\mathrm{Hg} / \mathrm{HgO}$, and the oxygen reduction current was measured to be -0.13 $\mathrm{mA}$; this is more than two times larger than that of the GQDs. ${ }^{23}$ This enhancement is mainly due to charge transfer behavior from GQD to electronegative FePC..$^{15,26}$

To obtain further insight into the electrocatalytic activity differences for the ORR on GQDs and GQD-FePC, LSV measurements on RDE system were performed in an $\mathrm{O}_{2}$-saturated $0.1 \mathrm{M} \mathrm{KOH}$ electrolyte at a scan rate of $20 \mathrm{mV} \mathrm{s}^{-1}$ with various rotating speeds from 400 to $2500 \mathrm{rpm}$. Fig. $4 \mathrm{a}-\mathrm{c}$ show the LSV polarization curves of GQDs, GQD-FePC, and $\mathrm{Pt} / \mathrm{C}$, respectively. The limiting current densities show a general increase with an increase in the rotating speed due to the improved diffusion of the electrolyte. ${ }^{28}$ To investigate the electrocatalytic efficiency of GQDs, GQD-FePC, and Pt/C, KouteckyLevich (K-L) plots were derived from the LSV polarization curves (from Fig. $4 \mathrm{a}-\mathrm{c}$ ). ${ }^{29}$ The linearity and parallelism of the K-L plots of Fig. $4 \mathrm{~d}-\mathrm{f}$ show a first-order reaction with regard to the dissolved oxygen and the electron transfer number for the ORR at each potential. ${ }^{30}$ For a clearer comparison between catalysts, the LSV curves at $1600 \mathrm{rpm}$ are presented in Fig. 5a. The onset potential of GQDs for the ORR was approximately $-0.13 \mathrm{~V} v s$. $\mathrm{Hg} / \mathrm{HgO}$, while a substantial positive shift to $-0.04 \mathrm{~V}$ vs. $\mathrm{Hg} /$ $\mathrm{HgO}$ was observed for GQD-FePC. This improved onset potential is attributed to the charge transfer, enhancing the first electron rate which is the main contributor for high onset potential. ${ }^{15,26}$ The limiting current of GQD-FePC for the ORR was calculated to be $-4.68 \mathrm{~mA} \mathrm{~cm}^{-2}$, which is 2.8 times higher than that of GQDs $\left(-1.65 \mathrm{~mA} \mathrm{~cm}^{-2}\right)$. These results show that the introduction of FePC into GQDs significantly enhances the offlimiting current density for the ORR compared to pure GQDs. These results agree with the CV analysis and further confirm that additional nitrogen can considerably improve the ORR catalytic activity. The number of transferred electrons is an important parameter related to the electrocatalytic activity for the ORR. The direct four-electron oxygen reduction process is known to be favorable due to its high efficiency and noncorrosive product. ${ }^{31}$ The number of transferred electrons per oxygen molecule related to the oxygen reduction of GQDs, GQDFePC, and $\mathrm{Pt} / \mathrm{C}$ is estimated by the $\mathrm{K}-\mathrm{L}$ equation ${ }^{32}$ shown below:
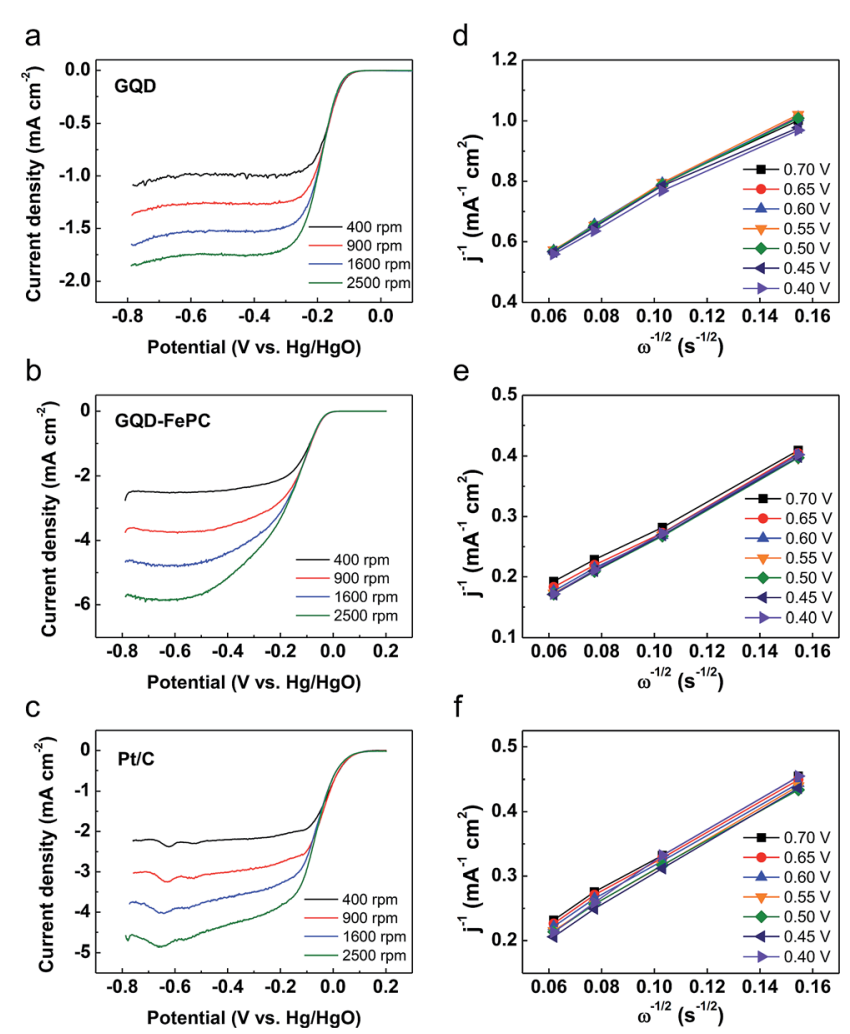

Fig. 4 LSV curves obtained by the RDE test for the ORR performance comparison of (a) GQDs, (b) GQD-FePC, and (c) Pt/C in $0.1 \mathrm{M} \mathrm{KOH}$ at a scan rate of $20 \mathrm{mV} \mathrm{s}^{-1}$ with various rotation rates from 400 to $2500 \mathrm{rpm}$. Koutecky-Levich plots of (d) GQDs, (e) GQD-FePC, and (f) $\mathrm{Pt} / \mathrm{C}$ at various potentials from -0.7 to $-0.4 \mathrm{~V}$ vs. $\mathrm{Hg} / \mathrm{HgO}$. 
a

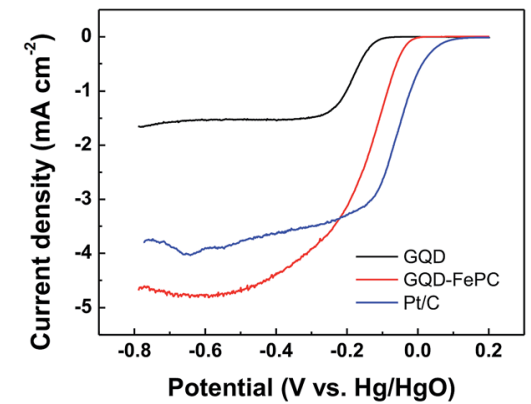

b

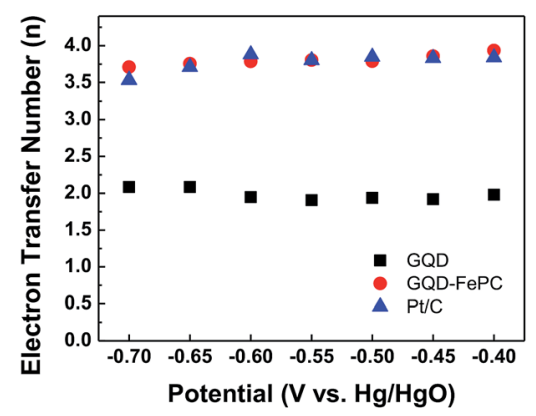

C

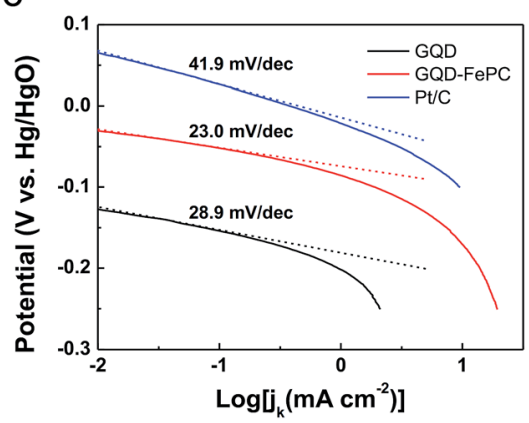

Fig. 5 (a) LSV, (b) the electron transfer number, and (c) Tafel plots for ORR of GQDs, GQD-FePC, and Pt/C, respectively. LSV was originated by Fig. $4 a-c$ with RDE rotation rates of $1600 \mathrm{rpm}$. The electron transfer numbers were defined at various potentials based on Koutecky-Levich plots. Tafel plots for ORR of each material in $0.1 \mathrm{M} \mathrm{O}_{2}$-saturated $\mathrm{KOH}$ electrolyte were derived from (a).

$$
\frac{1}{J}=\frac{1}{J_{\mathrm{L}}}+\frac{1}{J_{\mathrm{K}}}=\frac{1}{B \omega^{\frac{1}{2}}}+\frac{1}{J_{\mathrm{K}}}
$$

here, $J$ is the measured current density, and $J_{\mathrm{K}}$ and $J_{\mathrm{L}}$ are the kinetic and diffusion-limiting current densities, respectively. The diffusion-limiting current densities $\left(J_{\mathrm{L}}\right)$ can be expressed by the angular velocity of rotation $(\omega$ : $\omega=2 \pi N$, where $N$ is the linear rotation rate) and the Levich constant $(B)$, which can be determined from the slope of the K-L plots, which are based on the Levich equation:

$$
\begin{gathered}
B=0.62 n F C_{\mathrm{O}_{2}}\left(D_{\mathrm{O}_{2}}\right)^{2 / 3} v^{-1 / 6} \\
J_{\mathrm{K}}=n F k C_{\mathrm{O}_{2}}
\end{gathered}
$$

here, $n$ represents the overall number of transferred electrons in the ORR, $F$ is the Faraday constant $\left(F=96485 \mathrm{C} \mathrm{mol}^{-1}\right), C_{\mathrm{O}_{2}}$ is the bulk concentration of $\mathrm{O}_{2}$ in $0.1 \mathrm{M} \mathrm{KOH}\left(1.2 \times 10^{-6} \mathrm{~mol}\right.$ $\left.\mathrm{cm}^{-3}\right), D_{\mathrm{O}_{2}}$ is the diffusion coefficient in the KOH solution (1.9 $\left.\times 10^{-5} \mathrm{~cm}^{2} \mathrm{~s}^{-1}\right), v$ is the kinematic viscosity of the electrolyte $\left(0.01 \mathrm{~cm}^{2} \mathrm{~s}^{-1}\right)$, and $\mathrm{k}$ is the electron-transfer rate constant. According to eqn (1), and (2), the number of transferred electrons can be determined from the slope of the $\mathrm{K}-\mathrm{L}$ plots over the potential range from -0.40 to $-0.70 \mathrm{~V} v s$. $\mathrm{Hg} / \mathrm{HgO}$, as shown in Fig. $5 \mathrm{~b} .{ }^{32}$ While the average $n$ value for GQDs is calculated to be 1.98 , which is close to that of the classical two-electron transfer process, as has been shown by many other carbon-based materials such as carbon nanotube. ${ }^{33,34}$ GQD-FePC show a significant enhancement in their average $n$ value (3.80), which is similar to that of $\mathrm{Pt} / \mathrm{C}$ (average $n=3.77$ ). These results prove that GQD-FePC exhibit a dominant four-electron process for the ORR to reduce oxygen directly to $\mathrm{OH}^{-}$in alkaline electrolytes; ${ }^{35}$ alternatively, the GQDs have a two-electron transfer process that should yield $\mathrm{HO}_{2}{ }^{-33}$ Onset potential value and electron transfer number of GQD-FePC were compared to other previously reported works in Table S1. $\dagger$

Furthermore, using the calculated kinetic current density from the K-L equation, the Tafel plots for GQDs, GQD-FePC and $\mathrm{Pt} / \mathrm{C}$ catalysts were derived at low overpotentials of Fig. $5 \mathrm{a} .{ }^{36}$ As shown in Fig. 5c, the Tafel slope of Pt/C and GQDs were 41.9 and $28.9 \mathrm{mV} \mathrm{dec}^{-1}$, respectively, implying the higher inherent electrocatalytic activity of GQDs for ORR. After forming conjugates with FePC, the Tafel slope of GQDs was reduced to $23.0 \mathrm{mV} \mathrm{dec}{ }^{-1}$. This result reveals that the transfer of the first electron on both GQDs and GQD-FePC catalysts is the rate determining step under Temkin conditions for the adsorption of intermediates ${ }^{29}$ and the addition of FePC on GQD significantly influenced the overall ORR mechanism, and thus improved the electrocatalytic activity.

Due to the potential of GQD-FePC to be used as electrocatalysts for the ORR and replace commercial Pt/C catalysts, we further demonstrated the possible crossover of methanol, the carbon monoxide (CO) poisoning effect, and electrochemical stability, which are other challenges currently faced by Pt-based catalysts in fuel cells. ${ }^{19,37}$ Accordingly, chronoamperometric measurements were carried out at $-0.20 \mathrm{~V}$ in an $\mathrm{O}_{2}$-saturated $0.1 \mathrm{M} \mathrm{KOH}$ electrolyte at a rotation rate of $1600 \mathrm{rpm}$ for $1000 \mathrm{~s}$, and 3.0 $\mathrm{M}$ methanol and $\mathrm{CO}$ were then added into the electrolyte at $400 \mathrm{~s}$, and durability test of GQD-FePC, FePC, and Pt/C were carried out for $17000 \mathrm{~s}$. The corresponding chronoamperometric responses are shown in Fig. 6a-c respectively. In Fig. 6a, when GQD-FePC were exposed to methanol, the ORR current for GQD-FePC remained almost unchanged. However, a sharp decrease in the ORR current (by about 45\%) for Pt/C was observed, indicating that the electrochemical oxidation of methanol is preferred relative to the reduction of oxygen in the case of the $\mathrm{Pt} / \mathrm{C}$ catalysts.

To investigate the effect of $\mathrm{CO}$ on the electrocatalytic activity of GQD-FePC, 0.1 M KOH saturated with $\mathrm{CO}$ was injected into the electrolyte during the chronoamperometric measurements. As shown in Fig. 6b, the ORR current for Pt/C sharply decreased by about $20 \%$ as a result of $\mathrm{CO}$ poisoning. It shows that $\mathrm{CO}$ molecules were adsorbed onto the platinum surface. This resulted in a significant decrease in the number of 
a

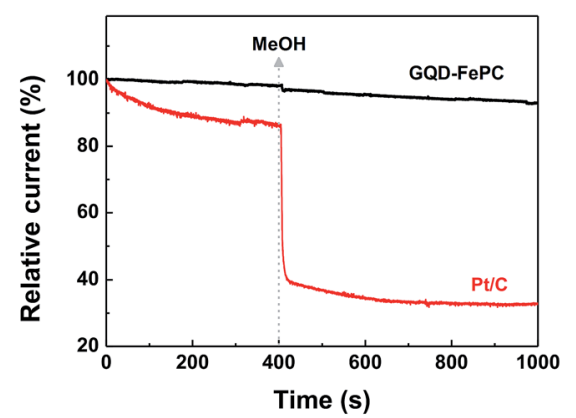

b

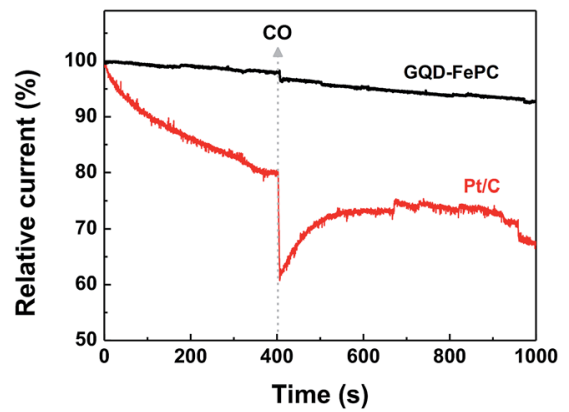

C

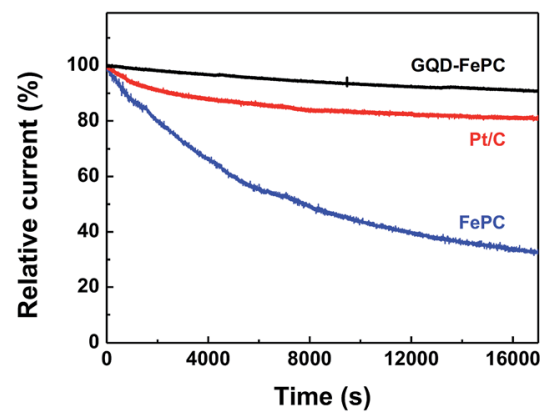

Fig. 6 The current-time chronoamperometric responses for the ORR of GQD-FePC and Pt/C in an $\mathrm{O}_{2}$-saturated $0.1 \mathrm{M} \mathrm{KOH}$ electrolyte at $-0.2 \mathrm{~V}$ versus $\mathrm{Hg} / \mathrm{HgO}$ at a rotation rate of $1600 \mathrm{rpm}$ : (a) methanol crossover test and (b) $\mathrm{CO}$ poisoning test upon the addition of $3.0 \mathrm{M}$ methanol and $\mathrm{CO}$ at around $400 \mathrm{~s}$. (c) Electrochemical stability of GQD-FePC, FePC, and Pt/C after $17000 \mathrm{~s}$.

electrocatalytic active sites for platinum due to the high chemical affinity of the $\mathrm{CO}$ molecule with platinum. ${ }^{6}$ Unlike $\mathrm{Pt} / \mathrm{C}$, the ORR current for GQD-FePC shows only a small decrease due to the reduced solubility of $\mathrm{O}_{2}$ in the electrolyte, which is caused by the diminished partial pressure of $\mathrm{O}_{2}$. This indicates that GQDFePC should have no reactivity with $\mathrm{CO}$ in nature.

The electrochemical stability of GQD-FePC, FePC, and Pt/C are shown in Fig. 6c. After the durability test for $17000 \mathrm{~s}$, FePC showed a significantly degraded current of $33.1 \%$ of its initial value during the measurement. After the hybridization with GQD, our GQD-FePC was able to retain $90.6 \%$ due to the synergistic effect by the covalent bonding between GQD and FePC. Furthermore, the value of $90.6 \%$ of GQD-FePC is definitely higher than that of $80.7 \%$ for commercial $\mathrm{Pt} / \mathrm{C}$ catalyst indicating the better electrochemical stability of GQD-FePC. The stability results of GQD-FePC were compared to other previously reported researches in Table $\mathrm{S} 2 \dagger$ and showed the higher stability value among them. Thus, since the electrocatalytic performance of GQD-FePC demonstrated excellent stability in terms of methanol crossover, CO poisoning effect, and electrocatalytic performance, their prospective potential for the replacement of commercial $\mathrm{Pt} / \mathrm{C}$ is expected.

\section{Conclusions}

In summary, we prepared iron phthalocyanine-functionalized graphene quantum dots via a facile ferric chloride reaction as a novel and efficient non-precious electrocatalyst. This successful functionalization not only prevents the aggregation of FePC, which can significantly decrease the activity, but also introduces additional nitrogen to GQDs, which can alter the electron structure of GQDs and modify the band gap. GQDFePC showed high electrocatalytic activity for the ORR in an alkaline electrolyte via the direct four-electron reaction. These possess an improved onset potential, a higher limiting current density than Pt/C, and notable methanol tolerance, insensitivity to $\mathrm{CO}$ poisoning and electrochemical stability. Thus, GQDFePC have the potential to be used as efficient non-precious electrocatalysts for the ORR and may be used in commercial applications of fuel cells.

\section{Conflict of interest}

The authors declare no competing financial interest.

\section{Acknowledgements}

K. H. Koh, S. H. Noh, T. H. Kim contributed equally to this work. This research was financially supported by Defense Acquisition Program Administration and Agency for Defense Development under the contract UD120080GD.

\section{Notes and references}

1 Z. Zhang, J. Zhang, N. Chen and L. Qu, Energy Environ. Sci., 2012, 5, 8869-8890.

2 D. Deng, L. Yu, X. Pan, S. Wang, X. Chen, P. Hu, L. Sun and X. Bao, Chem. Commun., 2011, 47, 10016-10018.

3 Y. Liu and P. Wu, ACS Appl. Mater. Interfaces, 2013, 5, 33623369.

4 Y. Li, Y. Zhao, H. Cheng, Y. Hu, G. Shi, L. Dai and L. Qu, J. Am. Chem. Soc., 2012, 134, 15-18.

5 G. Zhang, B. Y. Xia, X. Wang and X. W. David Lou, Adv. Mater., 2014, 26, 2408-2412.

6 L. Dai, Y. Xue, L. Qu, H. J. Choi and J. B. Baek, Chem. Rev., 2015, 115, 4823-4892.

7 X. Zhou, Z. Tian, J. Li, H. Ruan, Y. Ma, Z. Yang and Y. Qu, Nanoscale, 2014, 6, 2603-2607.

8 R. Baker, D. P. Wilkinson and J. Zhang, Electrochim. Acta, 2008, 53, 6906-6919.

9 H. Yang, Y. Zhang, F. Hu and Q. Wang, Nano Lett., 2015, 15, 7616-7620. 
10 S. J. Rowley-Neale, J. M. Fearn, D. A. C. Brownson, G. C. Smith, X. Ji and C. E. Banks, Nanoscale, 2016, 8, 14767-14777.

11 R. Zhang, C. Zhang and W. Chen, J. Mater. Chem. A, 2016, 4, 18723-18729.

12 A. B. Sorokin, Chem. Rev., 2013, 113, 8152-8191.

13 Z. Zhao, M. Li, L. Zhang, L. Dai and Z. Xia, Adv. Mater., 2015, 27, 6834-6840.

14 H. Wang, T. Maiyalagan and X. Wang, ACS Catal., 2012, 2, 781-794.

15 L. Qu, Y. Liu, J.-B. Baek and L. Dai, ACS Nano, 2010, 4, 13211326.

16 Y. Zheng, Y. Jiao, M. Jaroniec, Y. Jin and S. Z. Qiao, Small, 2012, 8, 3550-3566.

17 H. Park, S. H. Noh, J. H. Lee, W. J. Lee, J. Y. Jaung, S. G. Lee and T. H. Han, Sci. Rep., 2015, 5, 14163-14172.

18 M. C. Hsiao, S. H. Liao, M. Y. Yen, P. I. Liu, N. W. Pu, C. A. Wang and C. C. M. Ma, ACS Appl. Mater. Interfaces, 2010, 2, 3092-3099.

19 Y. Jiang, Y. Lu, X. Lv, D. Han, Q. Zhang, L. Niu and W. Chen, ACS Catal., 2013, 3, 1263-1271.

20 G. Dong, M. Huang and L. Guan, Phys. Chem. Chem. Phys., 2012, 14, 2557-2559.

21 M. Li, X. Bo, Y. Zhang, C. Han and L. Guo, Journal of J. Power Sources, 2014, 264, 114-122.

22 G. Yuan, G. Zhang, Y. Zhou and F. Yang, $R S C$ Adv., 2015, 5, 26132-26140.

23 R. Cao, R. Thapa, H. Kim, X. Xu, M. G. Kim, Q. Li, N. Park, M. Liu and J. Cho, Nat. Commun., 2013, 4, 2076-2083.
24 M.-S. Liao and S. Scheiner, J. Chem. Phys., 2001, 114, 97809791.

25 B. Zheng, C. Wang, X. Xin, F. Liu, X. Zhou, J. Zhang and S. Guo, J. Phys. Chem. C, 2014, 118, 7637-7642.

26 L. Zhang and Z. Xia, J. Phys. Chem. C, 2011, 115, 1117011176.

27 R. Burkitt, T. R. Whiffen and E. H. Yu, Appl. Catal., B, 2016, 181, 279-288.

28 H. Hu, L. Han, M. Yu, Z. Wang and X. W. David Lou, Energy Environ. Sci., 2016, 9, 107-111.

29 L. Cui, G. Lv and X. He, J. Power Sources, 2015, 282, 9-18.

30 R. Liu, D. Wu, X. Feng and K. Mullen, Angew. Chem., 2010, 49, 2565-2569.

31 M. Lefèvre and J.-P. Dodelet, Electrochim. Acta, 2003, 48, 2749-2760.

32 L. Lai, J. R. Potts, D. Zhan, L. Wang, C. K. Poh, C. Tang, H. Gong, Z. Shen, J. Lin and R. S. Ruoff, Energy Environ. Sci., 2012, 5, 7936-7942.

33 K. Gong, F. Du, Z. Xia, M. Durstock and L. Dai, Science, 2009, 323, 760-764.

34 S. Wang, D. Yu and L. Dai, J. Am. Chem. Soc., 2011, 133, 51825185.

35 B. Y. Guan, L. Yu and X. W. Lou, Energy Environ. Sci., 2016, 9, 3092-3096.

36 G. Zhang, W. Lu, F. Cao, Z. Xiao and X. Zheng, J. Power Sources, 2016, 302, 114-125.

37 S.-C. Yi, C. Y. Jung and W. J. Kim, Mater. Res. Bull., 2011, 46, 2433-2440. 